\title{
Adapting blood glucose meter biosensors for the measurement of lactose in dairy ingredients
}

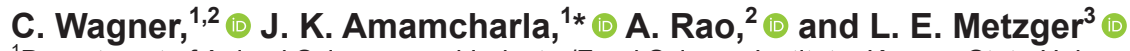 \\ ${ }^{1}$ Department of Animal Sciences and Industry/Food Science Institute, Kansas State University, Manhattan 66506 \\ ${ }^{2}$ Agropur Ingredients, Appleton, WI 54915 \\ ${ }^{3}$ Dairy Science Department, Midwest Dairy Foods Research Center, South Dakota State University, Brookings 57006
}

\begin{abstract}
Commonly used lactose assays [enzymatic spectrophotometric absorbance (EZA) and HPLC] for dairy ingredients are relatively expensive and time consuming. A blood glucose meter (BGM)-based method has successfully been documented as a rapid lactose assay in milk. However, the BGM-based method has not been evaluated in dairy ingredients. The objective of this study was to evaluate the BGM-based lactose analysis method in whey-derived (WD) and skim milk-derived (SMD) ingredients. The study was carried out in 4 phases. In phase 1 , the effect of $\mathrm{pH}$ and lactose concentrations on the BGM reading was investigated using a factorial design with 2 factors: $\mathrm{pH}(6.02-7.50)$ and lactose $(0.2$ or $0.4 \%)$. We found that BGM readings were significantly affected by lower $\mathrm{pH}$ values at both lactose levels. In phase 2, the effect of total solids and ingredient type was investigated using a factorial design with 2 factors: ingredient type (WD or SMD) and total solids (0-8\%). It was observed that the BGM reading was significantly affected by ingredient type and total solids. Phase 3 involved developing a linear relationship between the BGM reading and the EZA reference method to ascertain the accuracy of the proposed BGM method. Different ingredient types (WD or SMD) and non-lactose solids (0.5-27\%) model ingredient dilutions prepared over a range of lactose contents $(0.08-0.62 \%)$ were measured using the BGM and EZA methods. The average absolute percentage bias difference between the BGM method and EZA reference method results for these model dilutions was found to be between 2.2 and $7.3 \%$. In phase 4,15 samples procured from commercial sources ranging from 0.01 to $81.9 \%$ lactose were evaluated using the BGM method and EZA reference
\end{abstract}

\footnotetext{
Received November 12, 2019

Accepted March 28, 2020.

*Corresponding author: jayendra@ksu.edu
}

method. The average absolute percentage bias difference for lactose results between the 2 methods ranged from 3.6 to $5.0 \%$ and 5.3 to $9.7 \%$ for well-performing and poorly performing meters, respectively. Overall, the BGM method is a promising tool for rapid and low-cost analysis of lactose in both high-lactose and low-lactose dairy ingredients.

Key words: lactose, dairy ingredient, biosensor, rapid method, blood glucose meter

\section{INTRODUCTION}

Lactose, a disaccharide sugar comprising glucose and galactose, is the principal carbohydrate present in dairy products (Huber and BeMiller, 2017). Lactose is a ubiquitous constituent of dried dairy ingredients, making up $<2 \%$ in dry protein isolates and close to $100 \%$ in pure lactose powders (Huppertz and Gazi, 2016). Most common dairy ingredients are derived from either cheese whey or skim milk. The primary goal of protein isolate and concentrate manufacture is to remove lactose, fat, and minerals to fractionate the protein portion of the ingredient.

Routine analysis of lactose in dairy ingredients could lead to improved utilization of the ingredients as well as help correlate lactose concentration with the ingredient quality. For example, measuring the lactose content in permeate and retentate streams can be a valuable troubleshooting tool during the membrane concentration of dairy proteins (Olabi et al., 2015; Sluková et al., 2016). Likewise, incorporation of the correct amount of starting lactose can be a critical quality control tool for the optimization of whey fermentation (Sansonetti et al., 2010). Last, quality defects such as stickiness, caking, or browning in dairy powders are largely dependent on the amount and form of lactose present in dried ingredients (Olabi et al., 2015; Huppertz and Gazi, 2016; Gulzar and Jacquier, 2018).

Common methods of lactose analysis used by the dairy industry include HPLC (Upreti et al., 2006; Gabbanini et al., 2010) and enzymatic spectrophotometric 
absorbance assays (Lynch and Barbano, 2007). These established methods have a high level of sophistication and long analysis times that can be overcome by using biosensors, which are generally faster, simpler, and less expensive to use (Conzuelo et al., 2012; Glithero et al., 2013; Sharma and Leblanc, 2017). Few commercial biosensors for the measurement of lactose can be found in the current marketplace, largely due to the complexity of dairy product composition (Booth et al., 2017). Only recently, a commercial biosensor designed for lactose has been documented in the literature: the Biomilk 300 (Biolan Microbiosensores, Zamudio, Spain), which is tailored for the analysis of residual lactose in lactosefree dairy products (Churakova et al., 2019). Another lactose biosensor, Lactosens (Chr. Hansen Holding A/S, Hoersholm, Denmark), was also commercially launched with limited availability.

As a low-cost alternative to proprietary lactose biosensors, blood glucose meter (BGM) biosensors, usually associated with diabetes care, can be adapted to measure lactose in milk (Amamcharla and Metzger, 2011). First, lactose in a diluted sample is hydrolyzed using lactase enzyme, and the resulting glucose is measured using the BGM. Then, the BGM output is compared with a standard curve to measure the percentage of lactose in the original sample.

Any BGM is a possible candidate for measuring lactose as long as the enzymatic detection is based on glucose oxidase, glucose dehydrogenase-nicotinamide adenine dinucleotide, or glucose dehydrogenase-flavin adenine dinucleotide. Blood glucose meter sensors based on glucose dehydrogenase-pyrroloquinoline quinone cannot be used for lactose measurement because galactose can interfere with the glucose measurement (Schleis, 2007; Frias et al., 2010). Additionally, some sample matrix and environment-dependent interferences are known to cause bias in blood glucose measurements and may interfere in measurement of lactose in dairy ingredients. For example, a sample $\mathrm{pH}<6.8$ was shown to erroneously provide lower BGM output for glucose measurements in blood (Dungan et al., 2007). Varying hematocrit levels (volume of red blood cells relative to plasma volume in blood) have been shown to bias the BGM output for glucose measurements in blood on a hematocrit-proportional basis (Ramljak et al., 2013). The BGM measurement accuracy can also be affected by temperature and, to a lesser extent, humidity (Erbach et al., 2016). To create a robust BGMbased lactose measurement method, this work explored strategies to compensate for potential sample matrixdependent interferents such as $\mathrm{pH}$ and concentration of non-lactose solids in samples (similar to hematocrit effect on BGM output in blood).

\section{MATERIALS AND METHODS}

\section{Experimental Design}

The efficacy of the proposed BGM method was studied in 4 phases. In phase 1 , the effect of $\mathrm{pH}$ and lactose concentration on BGM output bias was investigated. In phase 2, BGM measurements were performed on several dairy ingredient types and total solids combinations to evaluate the effect of these parameters on the BGM output. The findings from phases 1 and 2 were applied to phase 3 , where a calibration procedure for quantification of lactose in model dairy ingredient dilutions was verified by analyzing the linear relationship of paired lactose results between the proposed BGM method and a reference method. In phase 4, the developed method was used to quantify the lactose content of commercial dairy ingredients to assess the robustness of the proposed method.

\section{Buffer, Dairy Ingredients, and Solution Preparation}

Phosphate-buffered saline was used as a diluent. A PBS buffer of $1 \times$ strength was prepared in 1-L batches from $10 \times$ PBS (Fisher Scientific, Pittsburgh, PA) and deionized water. Hydrochloric acid (1 N; Acros Organics, Pittsburgh, PA) was then used to adjust the $\mathrm{pH}$ ( $\mathrm{pH}=7$ unless otherwise indicated). A calibrated Accumet XL150 pH meter (Fisher Scientific) was used for all $\mathrm{pH}$ measurements. Then, ACS-certified grade $\alpha$-lactose monohydrate (Fisher Scientific) was used to adjust the lactose content of any model dilutions prepared. Whey-derived (WD) or skim milk-derived (SMD) ingredients described in this work were procured from Agropur Ingredients (Appleton, WI) and were obtained in dry form. All the powders were mixed with the titrated PBS diluent at room temperature using an overhead mixer equipped with a propeller-type spindle for $10 \mathrm{~min}$, and the resulting dispersions were stored between 1 and $4^{\circ} \mathrm{C}$ for at least $24 \mathrm{~h}$ to ensure a complete rehydration and equilibrium of $\alpha$ - and $\beta$-lactose anomers before any further analysis.

\section{Lactase Enzyme}

Enzeco lactase NL $\beta$-galactosidase or lactase enzyme used in this work was a gift from the Enzyme Development Corporation (New York, NY). According to the material specification data, the enzyme is prepared from a dairy yeast belonging to the Kluyveromyces genus and has an activity level between 2,450 and 3,150 yeast lactase units/g, with an optimum temperature between 31 and $40^{\circ} \mathrm{C}$ and solution $\mathrm{pH}$ between 6 and 7 . 


\section{BGM Measurements}

The BGM units used in this work include the ReliOn Prime (Walmart, Bentonville AR), Nova Max Plus (Nova Biomedical Corp., Waltham, MA), OneTouch Verio Flex (LifeScan Europe, Zug, Switzerland), and FreeStyle Precision Neo (Abbott Diabetes Care Ltd., Witney, UK). All the units were capable of measuring at least 20 to $500 \mathrm{mg} / \mathrm{dL}(0.02-0.5 \%)$ glucose in blood. To ensure proper functioning, new test strip lots were tested using the manufacturer-recommended control glucose solutions before any measurements.

No special sample preparation except dilution in PBS buffer was required for BGM lactose analysis. The BGM lactose analysis proposed in the current research involved weighing $5.0 \mathrm{~g}$ of diluted sample into a test tube, thoroughly mixing the sample with $0.1 \mathrm{~g}$ of lactase enzyme ( $\sim 245$ yeast lactase units), incubating the mixed sample at $40^{\circ} \mathrm{C}$ for approximately $15 \mathrm{~min}$, and then measuring the resulting glucose content by pipetting the incubated sample directly onto the capillary site of a BGM test strip. To maintain a consistent sample temperature, dilutions were removed from the incubation water bath just before the measurement and then promptly placed back into the water bath. All the BGM measurements were carried out in a temperaturecontrolled room $\left(22-24^{\circ} \mathrm{C}\right)$ with relative humidity measured between $<20$ and $39 \%$.

\section{Enzymatic Spectrophotometric Absorbance Reference Method}

An enzymatic spectrophotometric absorbance (EZA) assay was used as a reference method to quantify lactose in both model and commercial ingredient dilutions. Commercial lactose/D-galactose enzyme assay kits (RBiopharm AG, Darmstadt, Germany) provided all necessary reagents to carry out the analysis. Instructions provided with the kits detailed the assay preparation and calculations, with minor adaptations as recommended by Lynch and Barbano (2007). A DU-650 UVVIS spectrophotometer (Beckman Coulter, Brea, CA) was used to measure the absorbance of prepared assays at a wavelength of $340 \mathrm{~nm}$. Disposable acrylic cuvettes with a 1-cm path length were used. Each sample was measured in duplicate, with all results reported as the average of duplicates.

All samples destined for EZA analysis were first diluted with deionized water to adjust the lactose concentration to approximately $0.05 \%$ (wt/wt). Solutions containing only lactose or low amounts of whey solids $(\leq 3 \%)$ were filtered through $0.45-\mu \mathrm{m}$ nylon syringe filters and stored between 1 and $4^{\circ} \mathrm{C}$ for later use in the EZA assay. Samples containing caseins were also prepared using a similar method, except these solutions were centrifuged at $2,097 \times g$ for $10 \mathrm{~min}$ at room temperature (Savant high-speed microcentrifuge HSC10K, Thermo Scientific, Waltham, MA) before filtering. Samples containing higher amounts of whey solids $(<27 \%)$ were impossible to filter through syringe filters due to their viscosity; these solutions were first clarified via centrifugation at $8,410 \times g$ for $10 \mathrm{~min}$ and then filtered through $0.2-\mu \mathrm{m}$ nylon centrifuge filters with a 2 -mL capacity at $5,000 \times g$ for $15 \mathrm{~min}$ at room temperature (Legend XTR, Thermo Scientific).

\section{Phase 1}

The effect of solution $\mathrm{pH}$ and lactose concentration on BGM output bias was investigated in a $2 \times 6$ factorial design for 4 BGM brands. Model lactose solutions in PBS buffer with 0.2 and $0.4 \%$ (wt/wt) lactose concentrations were prepared at different $\mathrm{pH}$ levels (7.50, $7.00,6.73,6.53,6.27$, and 6.02) per the experimental design. The solutions were measured in a random order, in triplicate, using the proposed BGM method with 1 lot of test strips for each meter. The $\mathrm{pH}$ of each solution was measured again after assay preparation to ensure that great changes in buffered $\mathrm{pH}$ did not occur due to lactose and lactase addition. The BGM output measured for each sample was compared with the calculated glucose content of each lactose solution after hydrolysis (lactose \%, wt/wt, divided by 2) to calculate a percentage bias difference $\left(\mathbf{D}_{\mathbf{h}}\right)$ for each reading. The GLM procedure in SAS (Studio version, SAS Institute Inc., Cary, NC) was used for data analysis, treating $\mathrm{pH}$ and lactose concentration as class variables. Significant differences $(P<0.05)$ between experiment levels were detected using Tukey's honest significant difference test.

\section{Phase 2}

The effect of non-lactose solids amount and dairy ingredient type on BGM output was investigated using a $2 \times 5$ factorial experiment for 4 BGM brands. Model WD or SMD solutions at 5 total solids levels $(0,2,4,6$, or $8 \%$ ) were spiked with glucose monohydrate (Archer Daniels Midland, Chicago, IL) stock of known concentration to achieve $0.2 \%$ (wt/wt) glucose concentration directly measurable by the BGM biosensors. Whey protein isolate or micellar casein concentrate $(\geq 88 \%$ $\mathrm{db}$ protein) were used to generate the WD or SMD solutions, respectively, due to their individual purities in the primary non-lactose component in dairy ingredients - namely, protein. Each solution was measured in a random order, in triplicate, using a slight modification of the proposed BGM method where $0.1 \mathrm{~g}$ of deionized 
Table 1. One possible dilution scheme for lactose analysis of dairy ingredients via blood glucose meter, arranged by increasing dry-basis lactose content $^{1}$

\begin{tabular}{|c|c|c|c|c|}
\hline Example ingredient & $\begin{array}{l}\text { Estimated lactose } \\
\text { (\% dry basis })\end{array}$ & $\begin{array}{l}\text { Suggested } \\
\text { dilution factor }\end{array}$ & $\begin{array}{l}\text { Estimated post-hydrolysis } \\
\text { glucose in dilution }{ }^{2}(\mathrm{mg} / \mathrm{dL})\end{array}$ & $\begin{array}{l}\text { Approximate solids in dilution } \\
\text { from dairy ingredient }{ }^{3}(\%)\end{array}$ \\
\hline Whey protein isolate & 0.20 & 0.2857 & 30 & 26.9 \\
\hline Whey protein isolate & 2.00 & 0.2857 & 280 & 26.9 \\
\hline Whey protein concentrate 80 & 9.00 & 0.0327 & 150 & 3.08 \\
\hline Milk protein concentrate 70 & 18.00 & 0.0327 & 300 & 3.08 \\
\hline Whey protein concentrate 55 & 31.00 & 0.0053 & 80 & 0.50 \\
\hline Permeate & 84.00 & 0.0053 & 220 & 0.50 \\
\hline Lactose & 100.00 & 0.0053 & 260 & 0.50 \\
\hline
\end{tabular}

${ }^{1}$ The goal of any given dilution is to maintain a 0.03 to $0.3 \%(30-300 \mathrm{mg} / \mathrm{dL})$ solution glucose level after lactose hydrolysis (assuming $\sim 100 \%$ lactose hydrolysis), as this glucose range can be read by most meters.

${ }^{2}$ Calculated by dividing estimated diluted lactose percentage by 2 and multiplying by 1,000.

${ }^{3}$ Includes initial dilution, lactase addition, and assumed $4 \%$ moisture content of typical dried dairy ingredients.

water was added instead of $0.1 \mathrm{~g}$ of lactase enzyme such that the only analyte being measured was the glucose from the direct addition to the solutions (the dairy ingredients used as background solids are glucose free). The total solids content of each fully prepared dilution was verified using a Mark 3 LTE moisture analyzer (Sartorius AG, Goettingen, Germany). A percentage bias difference $\left(\mathbf{D}_{\mathbf{s}}\right)$ for each reading was calculated between the BGM output values and known glucose concentration directly spiked into each solution. The GLM procedure in SAS was used for data analysis for each meter separately, treating ingredient type and added solids target as class variables.

\section{Phase 3}

Phase 3 was designed to assess the accuracy of the proposed calibration procedure for lactose analysis in common dairy ingredients and was carried out in 2 parts. Calibration equations were developed between the actual lactose concentration and corresponding BGM output measured for a series of model dairy ingredient dilutions. Subsequently, validation of these calibration parameters was performed in the second part.

Calibration Procedure. Eighteen WD model calibration standards were developed as follows: powdered whey protein isolate was mixed with PBS buffer to create $0.5,3.0$, or $27 \%$ total whey solids slurries that represented powder dilution factors of $0.0053,0.0327$, and 0.2857 , respectively (Table 1 provides for further information on the selection of dilution schemes). The stock sample slurries were then partitioned into 6 samples each and spiked with lactose to achieve a final concentration between 0.08 and $0.62 \%$ (wt/wt) lactose. Similarly, 12 SMD model calibration standards were developed: micellar casein was mixed with PBS buffer to create 0.5 or $3.0 \%$ total skim milk solid slurries (a $27 \%$ solids solution was not considered due to high viscosity). The SMD slurries were also divided into 6 samples each and spiked with lactose to achieve a final concentration between 0.10 and $0.58 \%$ lactose. Each calibration solution was measured with the proposed BGM assay in a random order and in duplicate using the proposed BGM method with 3 different strip lots each for the Nova Max Plus and FreeStyle Precision Neo brands. Each solution was also analyzed for lactose using the EZA reference method. The glucose results from the BGM method were paired with the lactose results from the EZA method (in $\mathrm{mg} / \mathrm{dL}$ glucose and $\mathrm{mg} / \mathrm{dL}$ lactose, respectively) to develop linear calibration curves that can be used to convert BGM signals to percentage lactose that are specific for each solids combination, BGM brand, and BGM test strip lot.

Verification Procedure. Eighteen WD and 12 SMD independent verification standards were prepared as described in the calibration procedure. The independent verification samples were then measured using the proposed BGM lactose assay alongside the EZA reference lactose assay, with the BGM results (in $\mathrm{mg} /$ dL glucose) being converted to percentage lactose using Equation 1 in conjunction with the linear parameters (slope and intercept) derived in the calibration procedure:

$$
\% \text { lactose }=\frac{\text { meter reading }- \text { intercept }}{\text { slope } \times \mathrm{F}_{\mathrm{i}} \times 1,000},
$$

where $F_{i}$ is the product of dilution factors 1 to $\mathrm{i} . \mathrm{F}_{\mathrm{i}}$ is the product of all the dilutions performed through the measurement procedure. 
The calibration-adjusted BGM results and the EZA reference results (both in percentage wt/wt lactose) were then paired together and method comparison statistics between the 2 methods were carried out using the REG procedure in SAS to determine confidence intervals $(\alpha=0.05)$ for the resulting linear slope and intercept parameters. Microsoft Excel (Microsoft Corp., Redmond, WA) was used to calculate average absolute percentage bias difference (AD) and average bias difference (d) between the paired calibration-adjusted BGM lactose result and EZA reference method lactose result. A coefficient of variation was also calculated between duplicate BGM measurements, with results expressed as an average result for all 3 test strip lots used.

\section{Phase 4}

Lactose in a sampling of commercial dairy ingredients was measured in duplicate using both the proposed BGM method and the EZA reference method. Two lots of test strips each were used for the Nova Max Plus and FreeStyle Precision Neo BGM brands. Ingredients were mixed with buffer at dilution factors of $0.0053(0.5 \%$ total ingredient solids), 0.0327 (3.0\% total ingredient solids), and 0.2857 (27\% total ingredient solids) for 31 to $100,3.5$ to 18 , and $<2.0 \%$ lactose dry-basis ingredients, respectively (Table 1 provides further information on the selection of dilution schemes). The corresponding linear calibration curve parameters established in phase 3 were used to convert BGM outputs to percentage lactose in phase 4. Excel was used to calculate AD and d between each paired BGM-EZA lactose results, and a coefficient of variation was calculated between duplicate BGM measurements.

\section{RESULTS AND DISCUSSION}

\section{Phase 1}

Table 2 details the effect that $\mathrm{pH}$ and lactose concentration may have on BGM output bias, expressed as percentage bias difference $\left(D_{h}\right)$ between the calculated post-glucose content of the lactose-hydrolyzed solutions and the BGM output. The addition of lactase and lactose did not greatly alter the buffer $\mathrm{pH}(\leq 0.08 \mathrm{pH}$ unit shift after addition).

Effect of Lactose Concentration. At a given $\mathrm{pH}$ level, lactose concentration can have a significant effect on BGM output bias as observed by noncomparable $(P<0.05) \mathrm{D}_{\mathrm{h}}$ between the 2 lactose levels tested. The ReliOn Prime meter showed a strong positive bias associated with lactose concentration given that every result between the 0.2 and $0.4 \%$ lactose levels of the experiment was significantly $(P<0.05)$ different. On

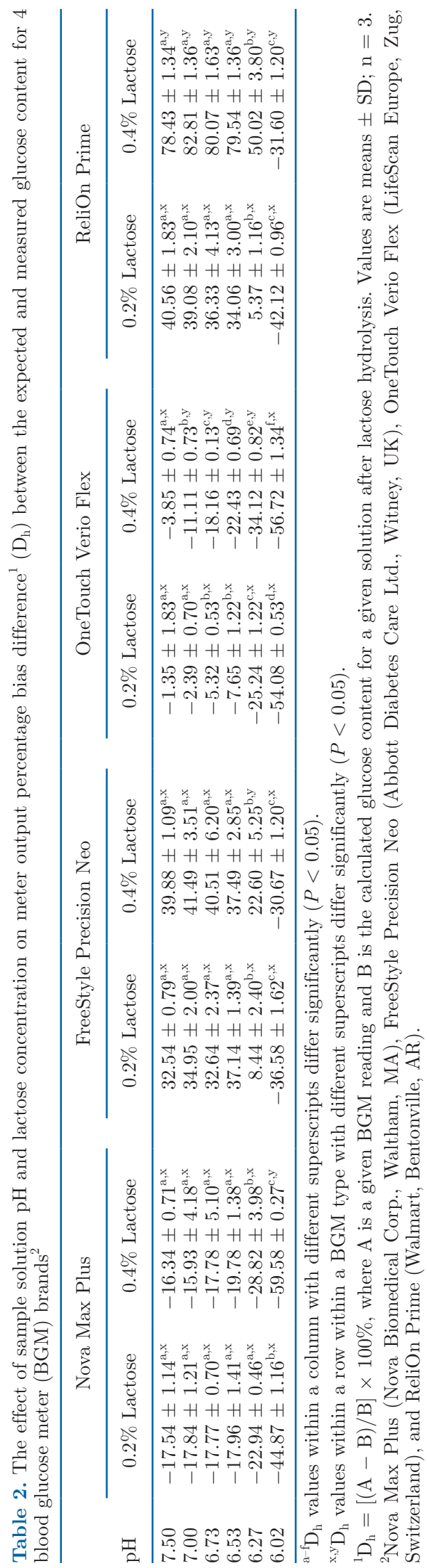


the other hand, the FreeStyle Precision Neo meter has a similar bias, albeit nonstatistically for most $\mathrm{pH}$ levels tested. Conversely, the OneTouch Verio Flex appears to have a negative bias associated with lactose content, with the trend being significant $(P<0.05)$ at all $\mathrm{pH}$ levels tested except 7.50 and 6.02. The Nova Max Plus meter was unique, showing no discernable $(P>0.05)$ lactose-proportional bias at any of the $\mathrm{pH}$ levels tested except the lowest $\mathrm{pH}$ level of 6.02. Because the BGM output biases were nonzero and dependent on lactose concentration for most meters, any BGM will need to have its output calibrated over a range of lactose concentrations to make useful quantifications. The need for calibration of the meter output was thus necessary and found to be consistent with other literature where the BGM method has been used for lactose quantification (Amamcharla and Metzger, 2011; Heinzerling et al., 2012; Churakova et al., 2019). The nonzero bias apparent in all of the meter outputs was likely due to material differences between the background matrices measured as opposed to what the meters were originally developed for (i.e., blood).

Effect of $\boldsymbol{p H}$. For all meters except the OneTouch Verio Flex, the BGM output biases were found to be comparable $(P>0.05)$ between the 7.50 and $6.53 \mathrm{pH}$ levels within a lactose level and BGM brand, indicating that these meters should provide accurate measurement within this $\mathrm{pH}$ range once a calibration is created to correct for the constant apparent measurement bias. The OneTouch Verio Flex meter specifically appears to be very $\mathrm{pH}$ sensitive, with a strong positive bias associated with $\mathrm{pH}$, indicating that this meter lacks measurement flexibility. Additionally, because the lowest $\mathrm{pH}$ level (6.02) always registered a significantly $(P<0.05)$ lower BGM output bias than any other $\mathrm{pH}$ level within a lactose level and BGM brand and the $\mathrm{pH}$ level of 6.27 showed a practical (albeit not always statistical) bias difference, it is recommended that measurements not be made at $\mathrm{pH}<6.53$. These results are consistent with reports showing unreliable BGM performance when measuring glucose in blood at $\mathrm{pH}<6.8$ due to changes in the activity level for the mediator enzymes in a test strip (Dungan et al., 2007). In addition, a reduction in lactase activity during incubation step of the BGM method hinders the hydrolysis of lactose into glucose and galactose and lowers the amount of glucose in the solution. Per the lactase enzyme manufacturer's specifications, the lactase has a $\mathrm{pH}$-dependent hydrolysis rate maximum between $\mathrm{pH} 6$ and 7 , and thus the finished dilution $\mathrm{pH}$ range for all further solution tested in this work was controlled to between 6.7 and 7.0 even though phase 1 results showed that no significant $\mathrm{pH}-$ dependent bias will be present in measurements utilizing some BGM sensors within the $\mathrm{pH}$ range of 6.53 to 7.50 .

\section{Phase 2}

Table 3 details the effect that different amounts or types of dairy ingredients can have on a given BGM brand's output bias, with bias being expressed as the percentage bias difference $\left(D_{s}\right)$ between the spiked glucose content $(0.2 \% \mathrm{wt} / \mathrm{wt})$ of each solution and the meter's output. Figure 1 illustrates the effect for each individual model dilution analyzed.

Effect of Ingredient Type. All meter output biases except for the ReliOn Prime meter were significantly ( $P$ $<0.05)$ affected by ingredient type. However, Figure 1 qualitatively indicates that the differences between similar-solids WD and SMD entries were relatively small on an individual basis, and it is worth noting that ingredient type contributed much less to the model error than did added solids amount (on average for all meters, 9.28 and $81.22 \%$ of the ANOVA model error were coming from solids type and amount, respectively). It is also impossible to ascertain whether the differences seen due to ingredient type are truly due to the differences in ingredient character or to slight dif-

Table 3. Statistical summary detailing the effect of skim milk- or whey-derived solids at different addition rates on percentage bias difference $\left(D_{\mathrm{s}}\right)$ between expected and measured glucose values for 4 blood glucose meter brands ${ }^{1}$ at a fixed, spiked glucose concentration of $0.2 \%$

\begin{tabular}{|c|c|c|c|c|c|c|c|c|c|}
\hline Source of error & df & \multicolumn{2}{|c|}{ Nova Max Plus } & \multicolumn{2}{|c|}{ FreeStyle Precision $\mathrm{Neo}$} & \multicolumn{2}{|c|}{ OneTouch Verio Flex } & \multicolumn{2}{|c|}{ ReliOn Prime } \\
\hline Ingredient type & 1 & 48.25 & $0.0317^{*}$ & 303.6 & $<0.0001^{*}$ & 68.39 & $0.0116^{*}$ & 40.68 & 0.2047 \\
\hline Type $\times$ amount & 4 & 79.62 & 0.1056 & 161.1 & $0.0129^{*}$ & 34.68 & 0.4414 & 91.48 & 0.4477 \\
\hline Model error & 9 & 388.5 & & 1,530 & & 2,361 & & 2,088 & \\
\hline Total error & 29 & 559.0 & & 1,724 & & 2,538 & & 2,562 & \\
\hline
\end{tabular}

${ }^{1}$ Nova Max Plus (Nova Biomedical Corp., Waltham, MA), FreeStyle Precision Neo (Abbott Diabetes Care Ltd., Witney, UK), OneTouch Verio Flex (LifeScan Europe, Zug, Switzerland), and ReliOn Prime (Walmart, Bentonville, AR).

${ }^{2}$ Mean sum of squares.

*Statistically significant at $P<0.05$. 
ferences in actual total solids content measured between corresponding WD and SMD dilution series (data not shown). All the same, it may conservatively be best practice to account for solids type during calibration and subsequent quantifications.
Effect of Added Solids Amount. All of the meter output biases were significantly $(P<0.05)$ affected by added solids amount. As detailed in Figure 1, increasing levels of solids for either ingredient type tested resulted in signal depression (decreasing $\mathrm{D}_{\mathrm{s}}$ ) for the

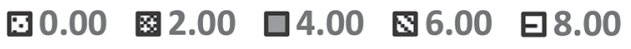

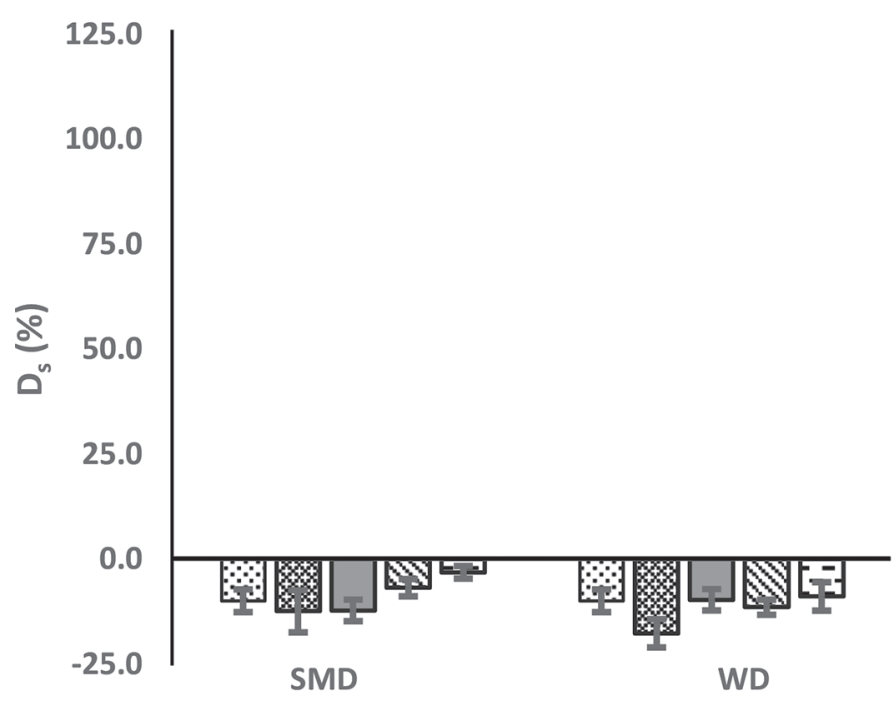

$\%$ Added solids

c

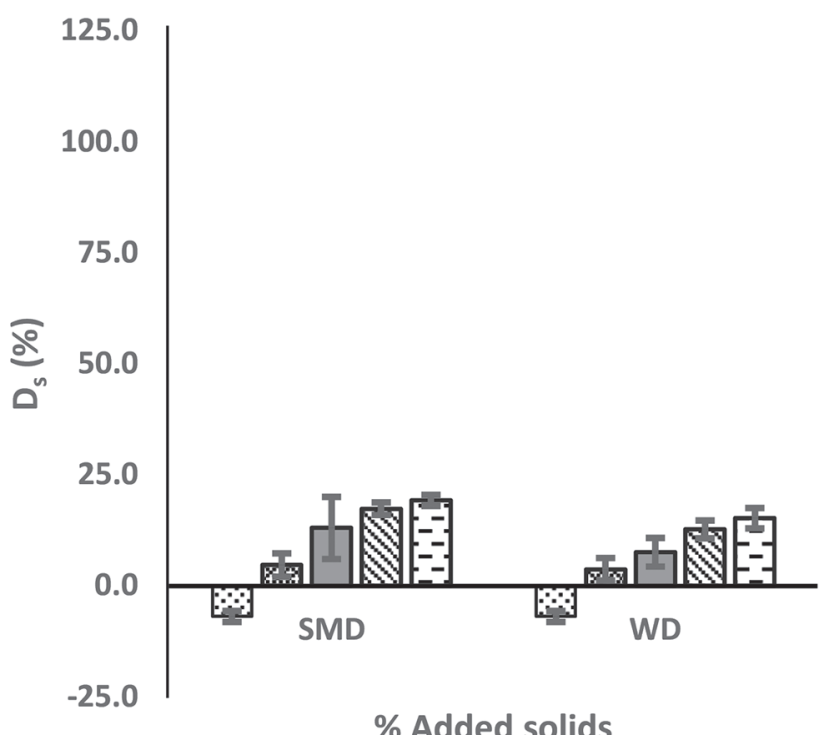

b

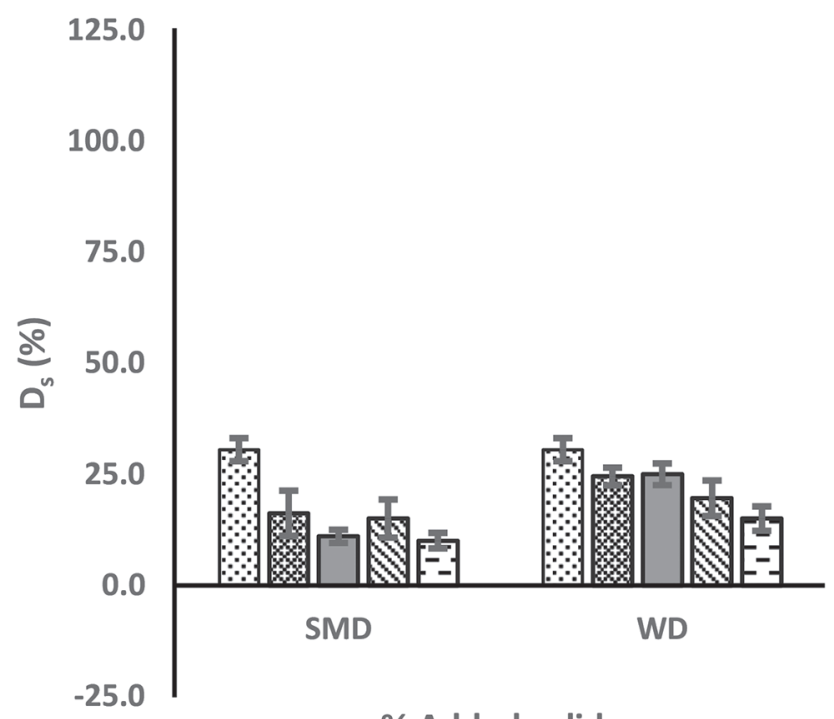

$\%$ Added solids

d

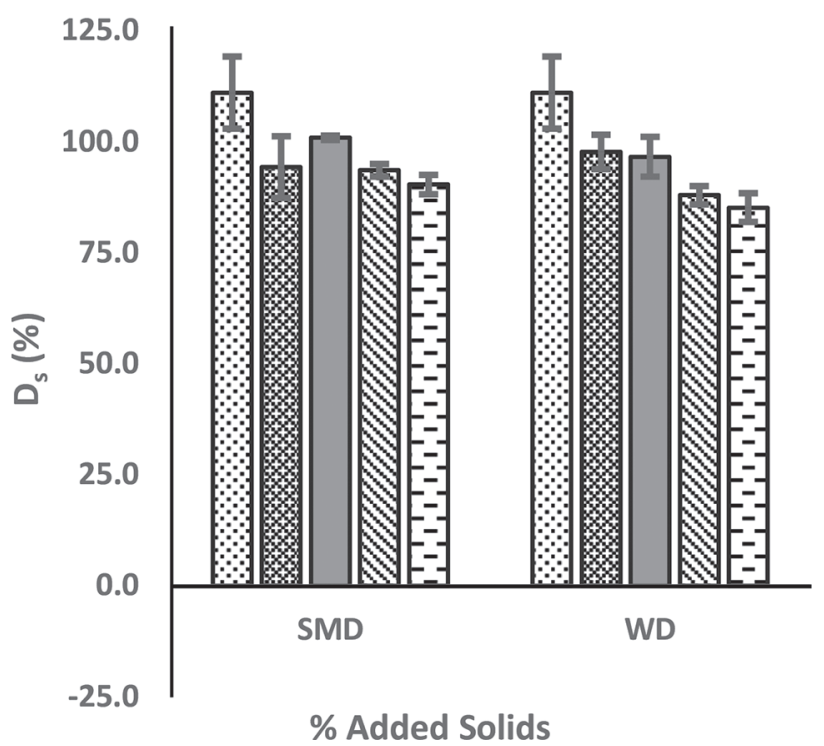

Figure 1. Effect of skim milk-derived (SMD) or whey-derived (WD) solids at different TS levels on percentage bias difference $\left(\mathrm{D}_{\mathrm{s}}\right)$ between expected and measured glucose values at a fixed, spiked glucose concentration of $0.2 \%$. Blood glucose meters: (a) Nova Max Plus (Nova Biomedical Corp., Waltham, MA); (b) FreeStyle Precision Neo (Abbott Diabetes Care Ltd., Witney, UK); (c) OneTouch Verio Flex (LifeScan Europe, Zug, Switzerland); (d) ReliOn Prime (Walmart, Bentonville, AR). $\mathrm{D}_{\mathrm{s}}=(\mathrm{A}-\mathrm{B}) / \mathrm{B} \times 100 \%$, where A is a given blood glucose meter reading and $\mathrm{B}$ is the fixed, spiked glucose concentration known for each test solution. Values are means; error bars represent $\mathrm{SD} ; \mathrm{n}=3$. 
FreeStyle Precision Neo meter and ReliOn Prime BGM brands or signal elevation (increasing $D_{s}$ ) for the Nova Max Plus and OneTouch Verio Flex BGM brands. This could be related to the analogous hematocrit effect in blood, where increasing levels of non-glucose solids may hinder glucose from getting to the enzyme test site. Intuitively, this explanation should universally translate into decreased BGM output; however, the Nova Max Plus and OneTouch Verio flex meters were both known to algorithmically adjust their outputs for hematocrit when measuring glucose in blood (Ramljak et al., 2013), so it is theorized that the positive relationship between $\mathrm{D}_{\mathrm{s}}$ and solids level observed for these meters is a result of the correction algorithm overcompensating for the presence of dairy ingredients. These results imply that the solids level of an ingredient dilution must be accounted for during calibration. The most common commercial dairy ingredients were therefore partitioned into 3 dilution schemes that controlled the total solids into a dilution while simultaneously keeping glucose concentrations between 0.03 and $0.3 \%$ (30-300 mg/ $\mathrm{dL}$ ) post-lactose hydrolysis, well within the measurable range for most BGM brands. To model these dilution schemes, total solids levels of $0.5,3.0$, and $27.0 \%$ were chosen to represent measurement-ready dilutions for 31 to $100,3.5$ to 18 , and $<2.0 \%$ lactose dry-basis ingredients, respectively. Assuming a moisture content of $4 \%$ for most dairy powders, the $0.5,3.0$, and $27.0 \%$ total solids translate into dilution factors of $0.0053,0.0327$, and 0.2857 , respectively.

Meters Selected for Further Testing. The Nova Max Plus and FreeStyle Precision Neo meters were selected for further testing in phases 3 and 4 . The Nova Max Plus promises robust performance due to its low and relatively constant bias over the range of lactose and solids levels tested. The OneTouch Verio Flex and ReliOn Prime meters were not used for further testing because they showed strong output bias dependence on background $\mathrm{pH}$ and solids amounts, respectively.

\section{Phase 3}

The calibration data are not shown but were of high quality. For example, none of the calibration curves had a coefficient of determination $\left(\mathbf{R}^{2}\right)$ value less than 0.986, indicating good linearity. Also, a limit of detection (LOD) was calculated for each calibration curve and test strip lot used (LOD $=3 \times$ RMSE/slope, where RMSE is the root mean squared error for a particular model) and averaged $0.037 \%(0.024-0.060 \%)$ lactose for the Nova Max Plus meter or $0.051 \%$ (0.025-0.062\%) lactose for the FreeStyle Precision Neo, well below the lactose levels measured in this work. Also, it was determined from the calibration data that test strip lot-specific calibration parameters should be used for subsequent lactose quantifications as evidenced by noncomparable $(P<0.05)$ slope parameters between some of the test strip lots used in the higher solid's samples. The paired method statistics between the EZA reference method and calibration-adjusted BGM method lactose results are shown in Tables 4 and 5 for the independently prepared verification WD and SMD model dilutions, respectively.

WD Ingredient Dilutions. The BGM method precision was found to be acceptable with an average coefficient of variation $\leq 4.93 \%$ for both of the meters studied. Accuracy in terms of agreement between the proposed BGM and EZA reference method was also good, with only 2 instances where regression slopes were significantly different from $1(3.0 \%$ WD model dilutions, FreeStyle Precision Neo, strip lot 2; 27\% WD model dilutions, Nova Max Plus, strip lot 2) and only 1 instance where intercepts were significantly different from 0 (27\% WD model dilutions, Nova Max Plus, strip lot 2). A slope and intercept not statistically different $(P>0.05)$ from 1 or 0 indicated good agreement between the 2 methods. Average bias difference (d) in lactose results between the methods was relatively small, with the average value of $d \leq 0.02 \%$ lactose in absolute value terms. Also, average bias difference did not trend high or low for any of the WD model dilutions, test strip lots, or meters tested, indicating that any differences observed between the test and reference methods were random. Examining the AD still invokes a favorable bias comparison between the BGM and EZA reference method given an average $\mathrm{AD}$ between 2.97 and $7.32 \%$. However, examining the range of $\mathrm{AD}$ for each average listed in Table 4 reveals some relatively high values; the highest absolute percentage bias differences $(\mathrm{AD}>15 \%)$ are universally associated with the lowest lactose concentrations measured $(<0.12 \%$ lactose). It is expected that there is a consistent amount of random error associated with the BGM measurement that is small in absolute terms but relatively high on a percentage difference basis when these errors occur at lower lactose concentrations.

SMD Ingredient Dilutions. Interpretations of the results for the SMD model dilutions were largely similar to those of the WD model dilutions. It can be concluded that the proposed dilution and ingredient type-based calibration schemes are likely to be successful in accurately predicting the lactose content of commercial dairy ingredient samples.

\section{Phase 4}

The as-is (wt/wt) lactose contents of 15 dry commercial dairy ingredients measured using the EZA 
Wagner et al.: DAIRY INDUSTRY TODAY

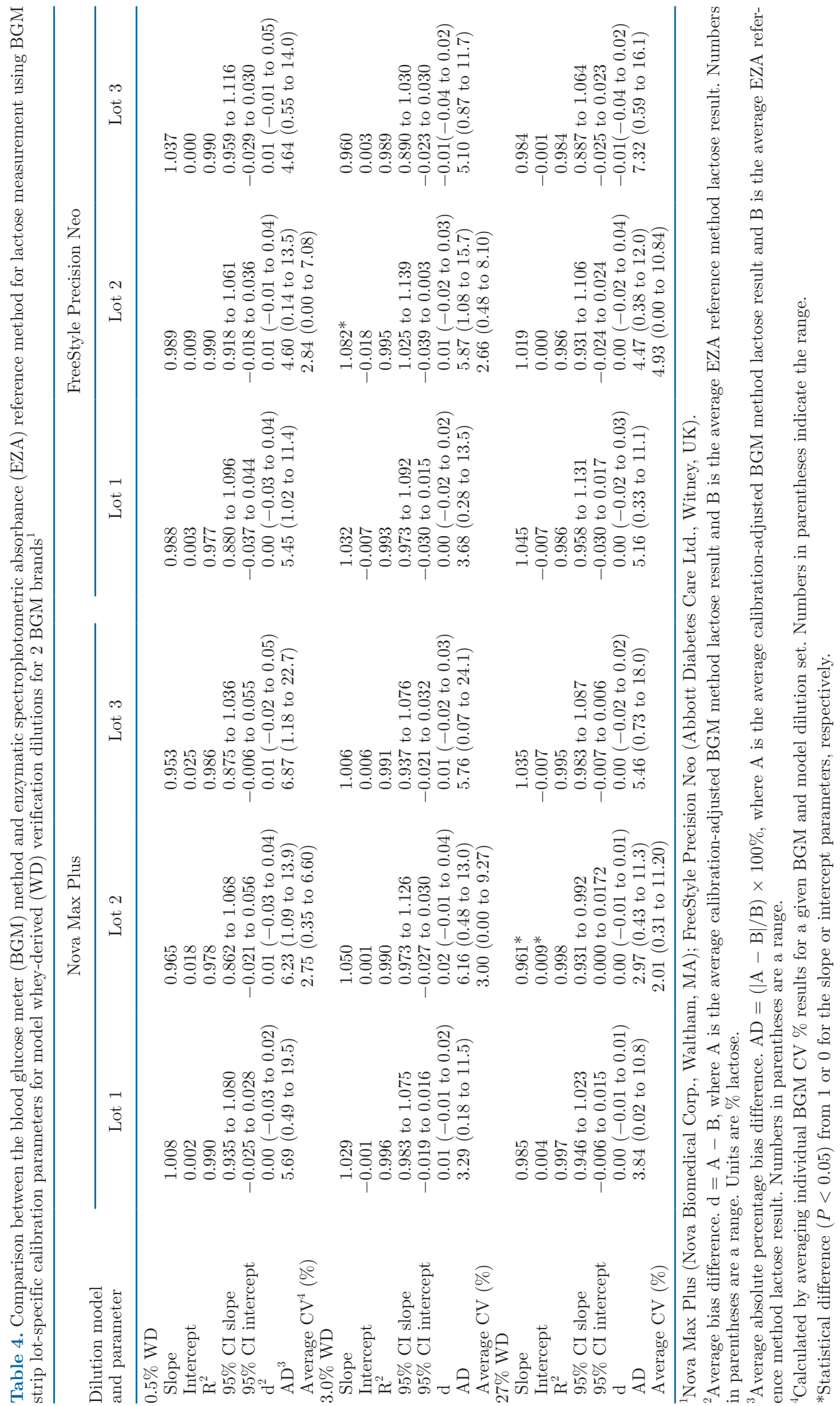


Wagner et al.: DAIRY INDUSTRY TODAY

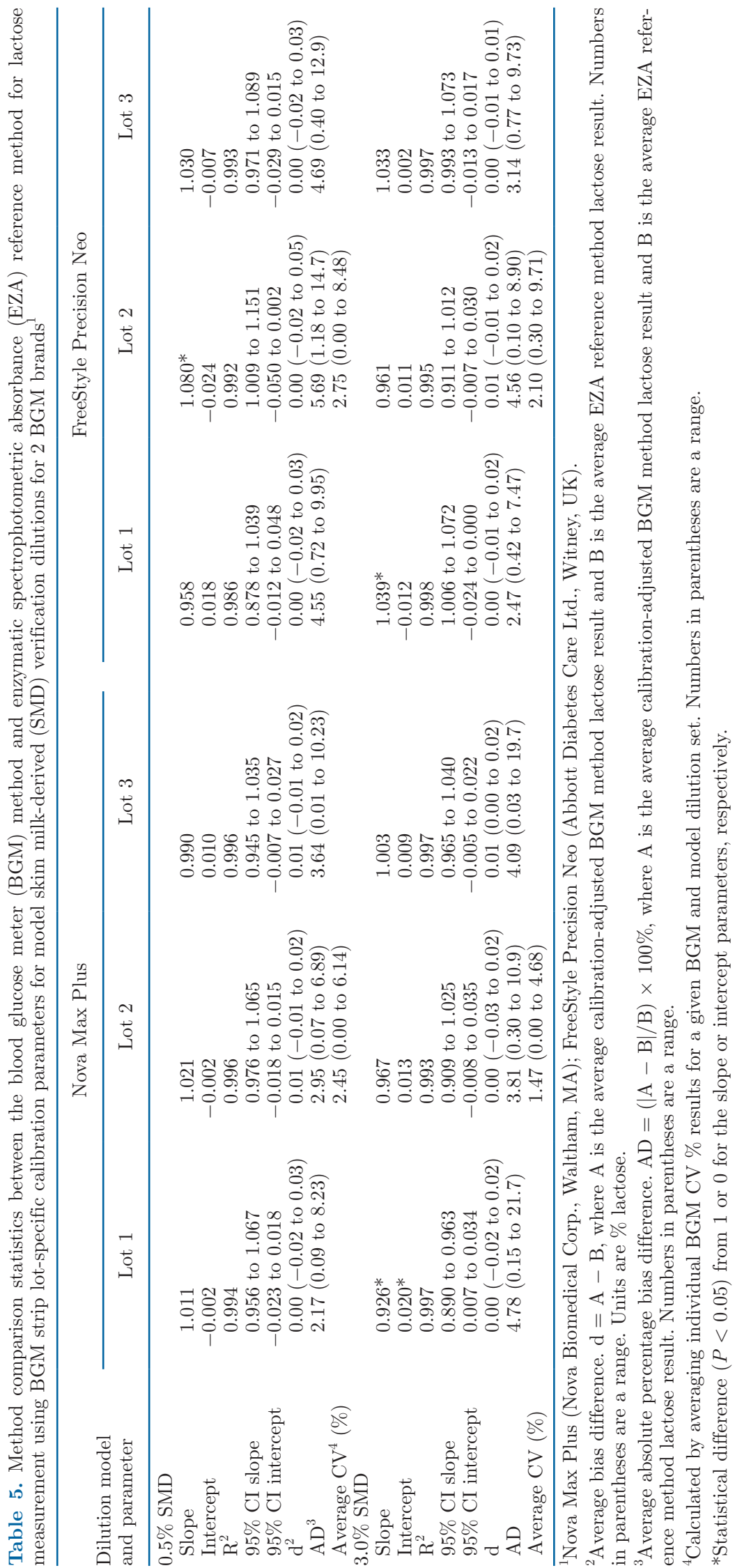


Table 6. Comparison of lactose concentration (\%) measured using the blood glucose meter (BGM) method and enzymatic spectrophotometric absorbance (EZA) reference method in commercial dairy ingredient samples using BGM strip lot-specific calibration parameters for the Nova Max Plus (Nova Biomedical Corp., Waltham, MA)

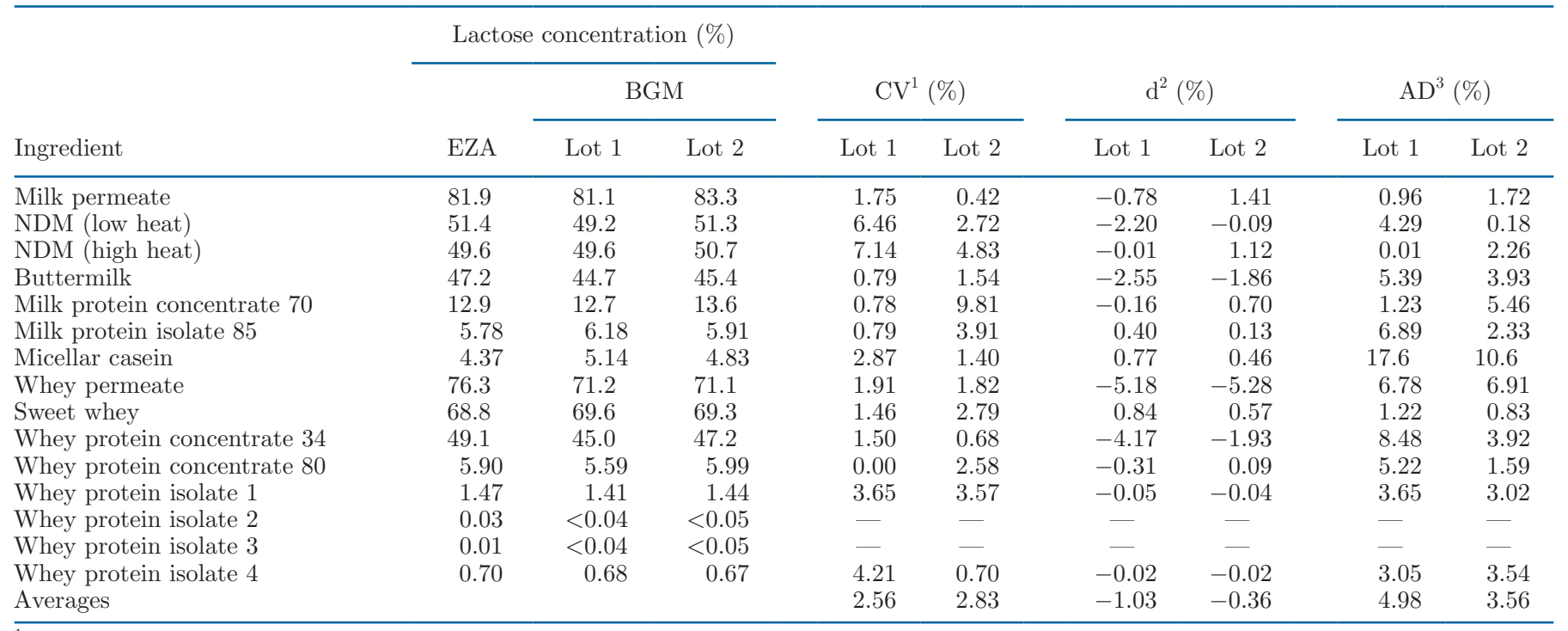

${ }^{1}$ Calculated between duplicate BGM measurements for each test strip lot. $\mathrm{n}=2$.

${ }^{2}$ Average bias difference. $\mathrm{d}=\mathrm{A}-\mathrm{B}$, where $\mathrm{A}$ is the average calibration-adjusted BGM method lactose result and B is the average EZA reference method lactose result. Units are \% lactose.

${ }^{3}$ Average absolute percentage bias difference. $\mathrm{AD}=(|\mathrm{A}-\mathrm{B}| / \mathrm{B}) \times 100 \%$, where $\mathrm{A}$ is the average calibration-adjusted BGM method lactose result and $\mathrm{B}$ is the average EZA reference method lactose result.

reference method and proposed BGM method with the modified calibration procedure are shown in Tables 6 and 7 for the Nova Max Plus and FreeStyle Precision Neo meter, respectively. The BGM method's precision was acceptable for both the Nova Max Plus and FreeStyle Precision Neo meters, with average coefficient of variation $<2.83 \%$ and $<3.54 \%$, respectively. Accuracy of the BGM method in terms of agreement between the BGM results and EZA results was found to depend on the meter used.

Nova Max Plus Accuracy. The Nova Max Plus BGM achieved relatively accurate results, with an average $\mathrm{AD}$ of 4.98 and $3.56 \%$ for test strip lots 1 and 2, respectively. One outlier was noted in the Nova Max Plus results when measuring lactose in micellar casein $(\mathrm{AD}=17.58$ and $10.60 \%$ for test strip lots 1 and 2 , respectively). The high error in terms of AD for micellar casein is consistent with observations in phase 3 where random BGM measurement error is inflated on a percentage basis at lower lactose concentrations; micellar casein was relatively low in lactose for the $3.0 \%$ SMD dilution-specific calibration series, measuring $0.14 \%$ lactose via the EZA method before adjusting for dilution (calibration range was $0.10-0.58 \%$ lactose). Thus, accurately measuring low amounts of lactose for a given calibration series may be a limitation of the BGM method. An alternative hypothesis for the high AD associated with this particular micellar casein ingredient could be the glycosylation of the $\kappa$-casein macropeptide portion of the protein. Glycosylated dairy proteins can be covalently linked to glucose, galactose (Recio et al., 2009), or lactose (Lillard et al., 2009), and the extent of glycosylation in $k$-casein macropeptide in particular can be difficult to predict because it depends largely on a cow's lactation cycle (O'Riordan et al., 2014). Although it is unlikely that these protein-attached carbohydrates could cause measurement inaccuracies with the proposed BGM lactose analysis, it must be mentioned as a possibility because proving otherwise is beyond the scope of this work.

FreeStyle Precision Neo Accuracy. The FreeStyle Precision Neo results showed a lower level of accuracy relative to the Nova Max Plus, with $\mathrm{AD}=5.32$ and $9.73 \%$ for test strip lots 1 and 2, respectively. A plausible explanation for the large amount of seemingly random error associated with the FreeStyle Precision Neo may relate to observations in phase 2 , where the FreeStyle Precision Neo was the only BGM with an output bias significantly $(P<0.05)$ affected by the interaction between the type and amount of added total solids (see Table 3). The FreeStyle Precision Neo showed acceptable accuracy in phase 3 when the SMD material used to create the calibration standards was the same as the independent verification samples measured, implying that poor performance of the FreeStyle Precision Neo with commercial samples may stem from 
material discrepancies between the commercial samples and the SMD ingredient chosen as background during calibration; this issue appears to be greatly exacerbated as higher levels of solids are incorporated (see high AD values associated with whey protein isolates 1 and 4 in Table 7). Thus, the FreeStyle Precision Neo may not be flexible enough to measure lactose in many different kinds of materials using the same model calibration parameters, which limits its practical use.

Blood glucose meter biosensors can be successfully adapted to measuring lactose in dairy ingredients if the following key concepts are adhered to:

1. A $1 \times$ PBS buffer should be used in dilutions as needed to control the final dilution $\mathrm{pH}$ to between 6.7 and 7.0.

2. Dilution schemes and corresponding calibration standards specific for a select range of dairy ingredients should be used. The composition of the material used in the calibration standards should loosely resemble the ingredients ultimately being measured (i.e., SMD vs. WD ingredients). Total diluted solids presence of $0.5,3.0$, and $27 \%$ can represent 31 to $100,3.5$ to 18 , and $<2.0 \%$ expected dry-basis lactose ingredients, respectively (these solids levels represent dilution factors of $0.005,0.0327$, or 0.2857 , respectively, for future commercial sample measurements). Pure lactose can be used to adjust the lactose concentration of the calibration standards anywhere from 0.08 to $0.6 \%$ as-is lactose.

3. A practical application of the method will use a BGM that is accurate, precise, and flexible (the Nova Max Plus worked well here).

\section{CONCLUSIONS}

A BGM can be used to routinely measure lactose in dairy ingredients. The suggested dilution factors are relevant to most dried dairy ingredients, but different dilution factors can be adapted to other dairy ingredients outside the scope of this work (e.g., liquid retentate) as long as the same concepts are applied. Once calibration curves have been developed, the advantages of the BGM method include achieving results in about 20 min, requiring no hazardous chemicals or expensive instrumentation, and requiring no sample preparation outside of sample dilution. Also, the method is low cost enough for routine use given a single test cost of less than US $\$ 0.50$ for the test strip and a one-time cost of approximately US $\$ 20$ for the BGM. It is not recommended that the BGM method be used to verify lactose absence in foods because lactose is not quantified directly with the method; thus, this method is more useful for applications such as process optimization or design, ingredient standardization, product develop-

Table 7. Comparison of lactose concentration (\%) measured using the blood glucose meter (BGM) method and enzymatic spectrophotometric absorbance (EZA) reference method in commercial dairy ingredient samples using BGM strip lot-specific calibration parameters for the FreeStyle Precision Neo (Abbott Diabetes Care Ltd., Witney, UK)

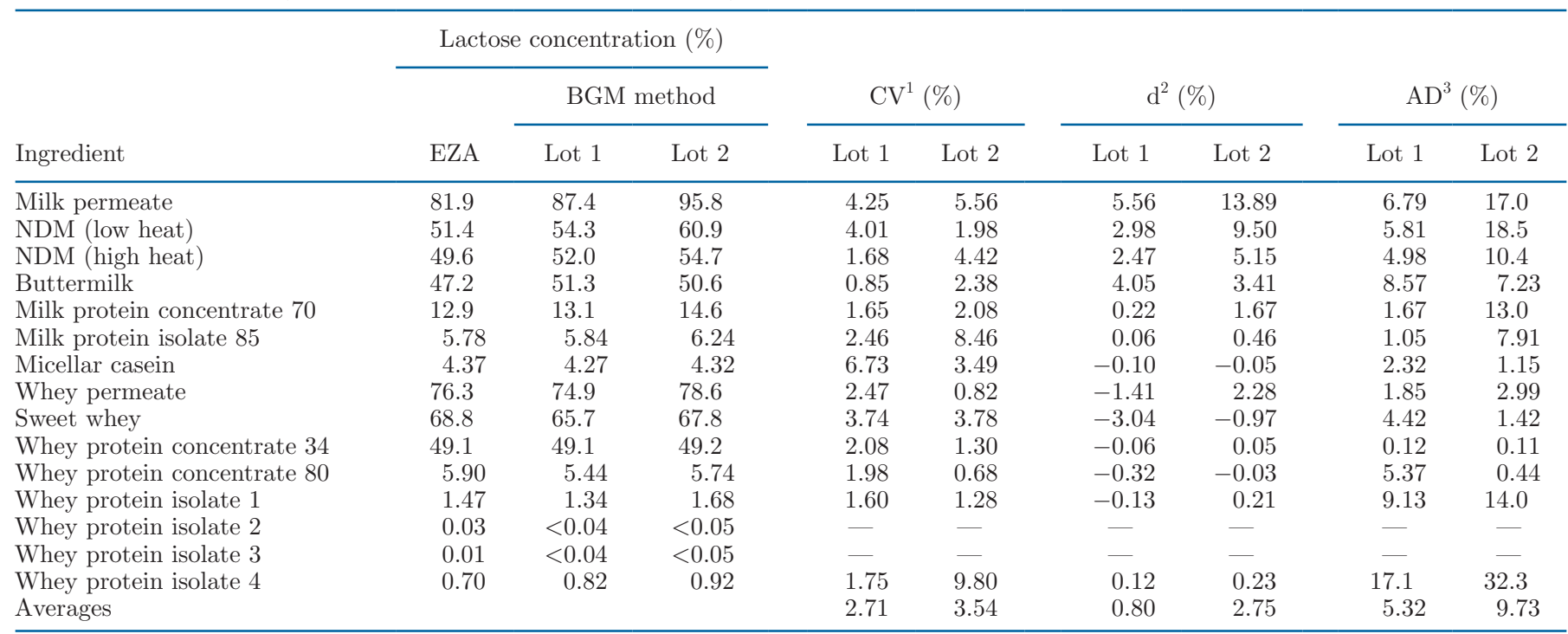

${ }^{1}$ Calculated between duplicate BGM measurements for each test strip lot. $\mathrm{n}=2$.

${ }^{2}$ Average bias difference. $\mathrm{d}=\mathrm{A}-\mathrm{B}$, where $\mathrm{A}$ is the average calibration-adjusted BGM method lactose result and B is the average EZA reference method lactose result. Units are \% lactose.

${ }^{3}$ Average absolute percentage bias difference. $\mathrm{AD}=(|\mathrm{A}-\mathrm{B}| / \mathrm{B}) \times 100 \%$, where $\mathrm{A}$ is the average calibration-adjusted BGM method lactose result and $\mathrm{B}$ is the average EZA reference method lactose result. 
ment, and routine lactose measurements for quality control purposes.

\section{ACKNOWLEDGMENTS}

This project was conducted under Kansas State Research and Extension contribution number 20-056-J. This work was partially supported by the USDA National Institute of Food and Agriculture (Washington, DC) Hatch project 1014344. We thank Midwest Dairy Foods Research Center (St. Paul, MN) for their partial financial support. We also thank Agropur Ingredients (Appleton, WI) for their support. Kansas State University (Manhattan) neither endorses nor takes responsibility for any products, goods, or services offered by outside vendors. The authors have not stated any conflicts of interest.

\section{REFERENCES}

Amamcharla, J. K., and L. E. Metzger. 2011. Development of a rapid method for the measurement of lactose in milk using a blood glucose biosensor. J. Dairy Sci. 94:4800-4809. https://doi.org/10 $.3168 /$ jds.2011-4416.

Booth, M. A., H. Karaosmanoglu, Y. Wu, and A. Partridge. 2017. Biosensor platforms for detecting target species in milk samples. Pages 71-103 in Food Biosensors. M. U. Ahmed, M. Zourob, and E. Tamiya, ed. Royal Society of Chemistry, Cambridge, UK.

Churakova, E., K. Peri, J. S. Vis, D. W. Smith, J. M. Beam, M. P. Vijverberg, M. C. Stor, and R. T. Winter. 2019. Accurate analysis of residual lactose in low-lactose milk: Comparing a variety of analytical techniques. Int. Dairy J. 96:126-131. https://doi.org/10 .1016/j.idairyj.2019.02.020.

Conzuelo, F., A. J. A. Reviejo, and J. M. J. Pingarron. 2012. Lactose in milk and dairy products: A focus on biosensors. Pages 549-567 in Dietary Sugars: Chemistry, Analysis, Function, Effects. V. Preedy, ed. Royal Society of Chemistry, Cambridge, UK.

Dungan, K., J. Chapman, S. S. Braithwaite, and J. Buse. 2007. Glucose measurement: Confounding issues in setting targets for inpatient management. Diabetes Care 30:403-409. https://doi.org/10 .2337/dc06-1679.

Erbach, M., G. Freckmann, R. Hinzmann, B. Kulzer, R. Ziegler, L. Heinemann, and O. Schnell. 2016. Interferences and limitations in blood glucose self-testing: An overview of the current knowledge. J. Diabetes Sci. Technol. 10:1161-1168. https://doi.org/10.1177/ 1932296816641433.

Frias, J. P., C. G. Lim, J. M. Ellison, and C. M. Montandon. 2010. Review of adverse events associated with false glucose readings measured by GDH-PQQ-based glucose test strips in the presence of interfering sugars. Diabetes Care 33:728-729. https://doi.org/ $10.2337 /$ dc09-1822.

Gabbanini, S., E. Lucchi, F. Guidugli, R. Matera, and L. Valgimigli. 2010. Anomeric discrimination and rapid analysis of underivatized lactose, maltose, and sucrose in vegetable matrices by U-HPLCESI-MS/MS using porous graphitic carbon. J. Mass Spectrom. 45:1012-1018. https://doi.org/10.1002/jms.1750.

Glithero, N., C. Clark, L. Gorton, W. Schuhmann, and N. Pasco. 2013. At-line measurement of lactose in dairy-processing plants. Anal. Bioanal. Chem. 405:3791-3799. https://doi.org/10.1007/s00216 -012-6598-y.

Gulzar, M., and J. C. Jacquier. 2018. Impact of residual lactose on dry heat-induced pre-texturization of whey proteins. Food Bioprocess
Technol. 11:1985-1994. https://doi.org/10.1007/s11947-018-2162 $-\mathrm{x}$.

Heinzerling, P., F. Schrader, and S. Schanze. 2012. Measurement of enzyme kinetics by use of a blood glucometer: Hydrolysis of sucrose and lactose. J. Chem. Educ. 89:1582-1586. https://doi.org/ 10.1021/ed200735f.

Huber, K. C., and J. N. BeMiller. 2017. Carbohydrates. Pages 91-170 in Fennema's Food Chemistry. 5th ed. D. Srinivasan and K. L. Parkin, ed. CRC Press, Boca Raton, FL.

Huppertz, T., and I. Gazi. 2016. Lactose in dairy ingredients: Effect on processing and storage stability. J. Dairy Sci. 99:6842-6851. https: //doi.org/10.3168/jds.2015-10033.

Lillard, J. S., D. Clare, and C. Daubert. 2009. Glycosylation and expanded utility of a modified whey protein ingredient via carbohydrate conjugation at low pH. J. Dairy Sci. 92:35-48. https://doi .org/10.3168/jds.2008-1263.

Lynch, J. M., and D. M. Barbano. 2007. Determination of the lactose content of fluid milk by spectrophotometric enzymatic analysis using weight additions and path length adjustment: Collaborative study. J. AOAC Int. 90:196-216.

O'Riordan, N., M. Kane, L. Joshi, and R. M. Hickey. 2014. Structural and functional characteristics of bovine milk protein glycosylation. Glycobiology 24:220-236. https://doi.org/10.1093/glycob/cwt162.

Olabi, A., S. Jinjarak, R. Jiménez-Flores, J. H. Walker, and H. Daroub. 2015. Compositional and sensory differences of products of sweet-cream and whey buttermilk produced by microfiltration, diafiltration, and supercritical $\mathrm{CO}_{2}$. J. Dairy Sci. 98:3590-3598. https://doi.org/10.3168/jds.2014-9141.

Ramljak, S., J. P. Lock, C. Schipper, P. B. Musholt, T. Forst, M. Lyon, and A. Pfützner. 2013. Hematocrit interference of blood glucose meters for patient self-measurement. J. Diabetes Sci. Technol. 7:179-189. https://doi.org/10.1177/193229681300700123.

Recio, I., F. J. Moreno, and R. Lopez-Fandino. 2009. Glycosylated dairy components: Their roles in nature and ways to make use of their biofunctionality in dairy products. Pages 170-211 in DairyDerived Ingredients: Food and Nutraceutical Uses. M. Corredig, ed. Woodhead Publishing, Boca Raton, FL.

Sansonetti, S., S. Curcio, V. Calabrò, and G. Iorio. 2010. Optimization of ricotta cheese whey (RCW) fermentation by response surface methodology. Bioresour. Technol. 101:9156-9162. https://doi.org/ 10.1016/j.biortech.2010.07.030.

Schleis, T. G. 2007. Interference of maltose, icodextrin, galactose, or xylose with some blood glucose monitoring systems. Pharmacotherapy 27:1313-1321. https://doi.org/10.1592/phco.27.9.1313.

Sharma, S. K., and R. M. Leblanc. 2017. Biosensors based on $\beta$-galactosidase enzyme: Recent advances and perspectives. Anal. Biochem. 535:1-11. https://doi.org/10.1016/j.ab.2017.07.019.

Sluková, M., A. Hinková, S. Henke, F. Smrž, M. Lukačíková, V. Pour, and Z. Bubník. 2016. Cheese whey treated by membrane separation as a valuable ingredient for barley sourdough preparation. J. Food Eng. 172:38-47. https://doi.org/10.1016/j.jfoodeng.2015.03 .023 .

Upreti, P., L. L. McKay, and L. E. Metzger. 2006. Influence of calcium and phosphorus, lactose, and salt-to-moisture ratio on Cheddar cheese quality: Changes in residual sugars and water-soluble organic acids during ripening. J. Dairy Sci. 89:429-443. https://doi .org/10.3168/jds.S0022-0302(06)72107-5.

\section{ORCIDS}

C. Wagner $\odot$ https://orcid.org/0000-0002-5682-7395

J. K. Amamcharla ๑ https://orcid.org/0000-0001-6620-0089

A. Rao (1) https://orcid.org/0000-0003-1332-3768

L. E. Metzger ๑ https://orcid.org/0000-0003-3929-4539 